Article

\title{
Fibonacci-Mann Iteration for Monotone Asymptotically Nonexpansive Mappings in Modular Spaces
}

\author{
Buthinah A. Bin Dehaish ${ }^{1,2}$ and Mohamed A Khamsi ${ }^{3,4, *}$ D \\ 1 Department of Mathematics, Faculty of Science For Girls, King Abdulaziz University, \\ Jeddah 21593, Saudi Arabia; bbindehaish@yahoo.com \\ 2 Department of Mathematics, Faculty of Science, University of Jeddah, Jeddah 23218, Saudi Arabia \\ 3 Department of Mathematical Sciences, The University of Texas at El Paso, El Paso, TX 79968, USA \\ 4 Department of Mathematics \& Statistics, King Fahd University of Petroleum and Minerals, \\ Dhahran 31261, Saudi Arabia \\ * Correspondence: mohamed@utep.edu
}

Received: 5 September 2018 ; Accepted: 8 October 2018; Published: 10 October 2018

check for updates

\begin{abstract}
In this work, we extend the fundamental results of Schu to the class of monotone asymptotically nonexpansive mappings in modular function spaces. In particular, we study the behavior of the Fibonacci-Mann iteration process, introduced recently by Alfuraidan and Khamsi, defined by

$$
x_{n+1}=t_{n} T^{\phi(n)}\left(x_{n}\right)+\left(1-t_{n}\right) x_{n},
$$

for $n \in \mathbb{N}$, when $T$ is a monotone asymptotically nonexpansive self-mapping.

Keywords: asymptotically nonexpansive mapping; Fibonacci sequence; fixed point; Mann iteration process; modular function spaces; monotone Lipschitzian mapping; opial condition; uniformly convexity
\end{abstract}

\section{Introduction}

Modular function spaces (MFS) find their roots in the study of the classical function spaces $L^{p}(\Omega)$ and their extensions by Orlicz and others. For more details on MFS, we recommend the book by Kozlowski [1]. Another interesting use of the modular structure, for whoever is looking for more applications, is the excellent book by Diening et al. [2] about Lebesgue and Sobolev spaces with variable exponents. Fixed point theory in MFS was initiated in 1990 in the original paper [3]. Since then this theory has become prevalent, culminating in the publication of the recent book by Khamsi and Kozlowski [4]. In this work, we continue investigating the fixed point problem in MFS. To be precise, we investigate the case of monotone mappings. This area of metric fixed point theory is new and attracted some attention after the publication of Ran and Reuring's paper [5]. An interesting reference with many applications of the fixed point theory of monotone mappings is the excellent book by Carl and Heikkilä [6]. We also suggest the work of Marin [7] for some applications associated to the iteration problem.

Since this work deals with the metric fixed point theory, we recommend the book by Khamsi and Kirk [8].

\section{Preliminaries}

For the basic definitions and properties of MFS, we refer the readers to the books $[1,4]$. Throughout this work, we assume the following: 
(i) $\Omega$ is a nonempty set;

(ii) $\Sigma$ is a nontrivial $\sigma$-algebra of subsets of $\Omega$;

(iii) $\mathcal{P}$ is a $\delta$-ring of subsets of $\Omega$ such that $A \cap B \in \mathcal{P}$ for any $A \in \mathcal{P}$ and $B \in \Sigma$;

(iv) there exists an increasing sequence $\left\{\Omega_{n}\right\}_{n \geq 1}$ in $\mathcal{P}$ such that $\Omega=\bigcup_{n \geq 1} \Omega_{n}$.

Consider the set $\mathcal{M}_{\infty}$ of extended measurable functions $f: \Omega \rightarrow[-\infty, \infty]$ such that there exists a sequence $\left\{f_{n}\right\}$ of simple functions whose supports are in $\mathcal{P}$ with $\left|f_{n}\right| \leq|f|$ and $\lim _{n \rightarrow \infty} f_{n}(t)=f(t)$, for any $t \in \Omega$.

Definition 1. ([1,4]) A convex and even function $\rho: \mathcal{M}_{\infty} \rightarrow[0, \infty]$ is called regular modular if

(i) $\rho(f)=0$ implies $f=0$;

(ii) $|f(t)| \leq|g(t)|$ for all $t \in \Omega$ implies $\rho(f) \leq \rho(g)$, where $f, g \in \mathcal{M}_{\infty}$ (we will say that $\rho$ is monotone);

(iii) $\left|f_{n}(t)\right| \uparrow|f(t)|$ for all $t \in \Omega$ implies $\rho\left(f_{n}\right) \uparrow \rho(f)$, where $f \in \mathcal{M}_{\infty}$ ( $\rho$ has the Fatou property).

Recall that $C \in \Sigma$ is said to be $\rho$-null if $\rho\left(f \mathbb{1}_{C}\right)=0$, for any simple function $f$ whose support is in $\mathcal{P}$, where $\mathbb{1}_{C}$ denotes the characteristic function of $C$. A property holds $\rho$-almost everywhere $(\rho$-a.e.) if the subset where it does not hold is $\rho$-null.

Remark 1. Let $\rho$ be convex regular modular. Let $f, g, h \in \mathcal{M}_{\infty}$ be such that $f \leq g \leq h \rho$-a.e. Then $0 \leq g-f \leq h-f$ and $0 \leq h-g \leq h-f \rho$-a.e., which imply

$$
\rho(g-f) \leq \rho(h-f) \text { and } \rho(h-g) \leq \rho(h-f) .
$$

Consider the set

$$
\mathcal{M}=\left\{f \in \mathcal{M}_{\infty} ;|f(t)|<\infty \rho-a . e\right\}
$$

The MFS $L_{\rho}$ is defined as

$$
L_{\rho}=\{f \in \mathcal{M} ; \rho(\lambda f) \rightarrow 0 \text { as } \lambda \rightarrow 0\}
$$

The following theorem is essential throughout this work.

Theorem 1 ([1,4]). Let $\rho$ be convex regular modular.

(1) If $\rho\left(\beta h_{n}\right) \rightarrow 0$, for some $\beta>0$, then there exists a subsequence $\left\{h_{\psi(n)}\right\}$ such that $h_{\psi(n)} \rightarrow 0 \rho-$ a.e.

(2) If $h_{n} \rightarrow h \rho-$ a.e., then $\rho(h) \leq \liminf _{n \rightarrow \infty} \rho\left(h_{n}\right)$.

The following definition mimics the metric properties using the modular.

Definition $2([1,4])$. Let $\rho$ be convex regular modular.

(1) $\left\{g_{n}\right\}$ is said to $\rho$-converge to $g$ if $\lim _{n \rightarrow \infty} \rho\left(g_{n}-g\right)=0$.

(2) A sequence $\left\{g_{n}\right\}$ is called $\rho$-Cauchy if $\lim _{n, m \rightarrow \infty} \rho\left(g_{n}-g_{m}\right)=0$.

(3) A subset $C$ of $L_{\rho}$ is said to be $\rho$-closed if for any sequence $\left\{g_{n}\right\}$ in $C \rho$-convergent to $g$ implies that $g \in C$.

(4) A subset $A$ of $L_{\rho}$ is called $\rho$-bounded if its $\rho$-diameter

$$
\delta_{\rho}(A)=\sup \{\rho(g-h) ; g, h \in A\}
$$

is finite.

Note that, despite the fact that $\rho$ does not satisfy the triangle inequality in general, the $\rho$ limit is unique. However, the $\rho$-convergence may not imply $\rho$-Cauchy behavior. Despite this setback, 
we can prove that any $\rho$-Cauchy sequence in $L_{\rho}$ is $\rho$-convergent, i.e., $L_{\rho}$ is $\rho$-complete $[1,4]$. Moreover, the Fatou property will show that the $\rho$-balls $B_{\rho}(f, r)=\left\{g \in L_{\rho} ; \rho(f-g) \leq r\right\}$ are $\rho$-closed.

The following result will be used throughout:

Theorem 2 ([1,4]). Let $\rho$ be convex regular modular. Let $\left\{g_{n}\right\} \subset L_{\rho}$ be a sequence which $\rho$-converges to $g$. The following hold:

(i) if $\left\{g_{n}\right\}$ is monotone increasing, i.e., $g_{n} \leq g_{n+1} \rho$-a.e., for any $n \geq 1$, then $g_{n} \leq g \rho$-a.e., for any $n \geq 1$.;

(ii) if $\left\{g_{n}\right\}$ is monotone decreasing, i.e., $g_{n+1} \leq g_{n} \rho$-a.e., for any $n \geq 1$, then $g \leq g_{n} \rho$-a.e., for any $n \geq 1$.

Next we discuss a property called uniform convexity, which plays an important part in metric fixed point theory.

Definition 3 ([4]). Let $\rho$ be convex regular modular. Let $r>0$ and $\varepsilon>0$. Consider the following set:

$$
D(r, \varepsilon)=\left\{(f, g) ; f, g \in L_{\rho}, \rho(f) \leq r, \rho(g) \leq r, \rho(f-g) \geq \varepsilon r\right\} .
$$

Then define

$$
\delta_{\rho}(r, \varepsilon)=\inf \left\{1-\frac{1}{r} \rho\left(\frac{f+g}{2}\right) ;(f, g) \in D(r, \varepsilon)\right\} .
$$

(i) $\rho$ is said to be uniformly convex (UC) if for every $r>0$ and $\varepsilon>0$, we have $\delta_{\rho}(r, \varepsilon)>0$.

(ii) $\rho$ is said to be (UUC) if for every $s \geq 0, \varepsilon>0$ there exists $\eta(s, \varepsilon)>0$ such that $\delta_{\rho}(r, \varepsilon)>\eta(s, \varepsilon)>0$, for $r>s$.

Example 1. As an example of modular function spaces, we consider the Orlicz spaces. These spaces were introduced by Orlicz and Birnbaum [9]. The function space is defined as follows:

$$
L^{\varphi}=\left\{f: \mathbb{R} \rightarrow \mathbb{R} ; \exists \lambda>0: \int_{\mathbb{R}} \varphi(\lambda|f(x)|) d m(x)<\infty\right\}
$$

where $\varphi:[0, \infty) \rightarrow[0, \infty)$ is assumed to be a convex function which is increasing to infinity. In other words, $\varphi$ behaves similarly to the power functions $\varphi(t)=t^{p}, p \geq 1$. The functional $\rho: L^{\varphi} \rightarrow[0, \infty)$ defined by

$$
\rho(f)=\int_{\mathbb{R}} \varphi(|f(x)|) d m(x)
$$

is convex regular modular. In this case, the uniform convexity of the modular was investigated in [10,11]. For example, the Orlicz functions that will generate a uniformly convex modular, one may take $\varphi_{1}(t)=$ $e^{|t|}-|t|-1$ and $\varphi_{2}(t)=e^{t^{2}}-1[12,13]$.

Modular functions which are uniformly convex enjoy a property similar to reflexivity in Banach spaces.

Theorem 3 ([4,10]). Let $\rho$ be (UUC) convex regular modular. Then $L_{\rho}$ has the property $(R)$, i.e., every sequence $\left\{C_{n}\right\}$ of nonempty, $\rho$-bounded, $\rho$-closed, convex subsets of $L_{\rho}$ such that $C_{n+1} \subset C_{n}$, for any $n \in \mathbb{N}$, has a nonempty intersection, i.e., $\bigcap_{n \in \mathbb{N}} C_{n} \neq \varnothing$.

Remark 2. Let $\rho$ be (UUC) convex regular modular. Let $K$ be a $\rho$-bounded convex $\rho$-closed nonempty subset of $L_{\rho}$. Let $\left\{f_{n}\right\} \subset K$ be a monotone increasing sequence. Since order intervals in $L_{\rho}$ are convex and $\rho$-closed, then the property $(R)$ implies

$$
\bigcap_{n \geq 1}\left\{f \in K ; f_{n} \leq f \rho-\text { a.e. }\right\} \neq \varnothing .
$$


In other words, there exists $f \in K$ such that $f_{n} \leq f \rho$-a.e., for any $n \geq 1$. A similar conclusion holds for decreasing sequences.

The following lemma is useful throughout this work.

Lemma 1 ([14]). Let $\rho$ be $(U U C)$ convex regular modular. Let $R>0$ and $\left\{\alpha_{n}\right\} \subset[a, b]$ with $0<a \leq b<1$. Let $\left\{f_{n}\right\}$ and $\left\{g_{n}\right\}$ be in $L_{\rho}$. Assume that

$$
\left\{\begin{array}{l}
\limsup _{n \rightarrow \infty} \rho\left(f_{n}\right) \leq R, \\
\limsup _{n \rightarrow \infty} \rho\left(g_{n}\right) \leq R, \\
\lim _{n \rightarrow \infty} \rho\left(\alpha_{n} f_{n}+\left(1-\alpha_{n}\right) g_{n}\right)=R .
\end{array}\right.
$$

Then $\lim _{n \rightarrow \infty} \rho\left(f_{n}-g_{n}\right)=0$ holds.

The concept of $\rho$-type function is fundamental in investigating the existence of fixed points. First let us recall the definition of a modular type function.

Definition 4 ([4]). Let $\rho$ be convex regular modular. Let $K$ be a nonempty subset of $L \rho$. The function $\varphi: K \rightarrow[0, \infty]$ is said to be a $\rho$-type if there exists a sequence $\left\{h_{m}\right\}$ in $L_{\rho}$ such that

$$
\varphi(f)=\limsup _{m \rightarrow \infty} \rho\left(h_{m}-f\right),
$$

for any $f \in K$. A sequence $\left\{f_{n}\right\}$ in $K$ is called a minimizing sequence of $\varphi$ if $\lim _{n \rightarrow \infty} \varphi\left(f_{n}\right)=\inf \{\varphi(f) ; f \in K\}$.

We have the following amazing result about $\rho$-type functions in MFS.

Lemma 2 ([14]). Let $\rho$ be (UUC) convex regular modular. Then any minimizing sequence of any $\rho$-type defined on a $\rho$-bounded $\rho$-closed convex nonempty subset $C$ of $L_{\rho}$ is $\rho$-convergent. Its $\rho$-limit is independent of the minimizing sequence.

Before we finish this section, we give the modular definitions of monotone Lipschitzian mappings.

Definition 5. Let $\rho$ be convex regular modular. Let $C$ be nonempty subset of $L_{\rho}$. Let $T: C \rightarrow C$ be a mapping.

(i) $T$ is said to be monotone if $f \leq g \rho$-a.e. implies $T(f) \leq T(g) \rho$-a.e., for any $f, g \in C$.

(ii) $T$ is called monotone asymptotically nonexpansive (in short $M-A-N$ ) if $T$ is monotone and there exists $\left\{k_{n}\right\}$, with $k_{n} \geq 1$ for any $n \geq 1$ such that $\lim _{n \rightarrow \infty} k_{n}=1$ and

$$
\rho\left(T^{n}(g)-T^{n}(h)\right) \leq k_{n} \rho(g-h),
$$

for any $g$ and $h$ in $C$ such that $g \leq h \rho$-a.e., and $n \geq 1$.

(iii) $g$ is a fixed point of $T$ whenever $T(g)=g$.

The fixed point theory for asymptotically nonexpansive mappings finds its root in the work of Goebel and Kirk [15]. Following the success of the fixed point theory of monotone mappings, an existence fixed point theorem for M-A-N mappings was proved in [16] and its modular version in [17]. Before we state the main result of [17], recall that a map $T$ is said to be $\rho$-continuous if $\left\{g_{n}\right\}$ $\rho$-convergent to $g$ implies $\left\{T\left(g_{n}\right)\right\} \rho$-convergent to $T(g)$. 
Theorem 4 ([17]). Let $\rho$ be (UUC) convex regular modular. Let $C$ be a $\rho$-bounded $\rho$-closed convex nonempty subset of $L_{\rho}$. Let $T: C \rightarrow C$ be a $\rho$-continuous $M-A-N$ mapping. Assume there exists $f_{0} \in K$ such that $f_{0} \leq T\left(f_{0}\right)$ (resp. $\left.T\left(f_{0}\right) \leq f_{0}\right) \rho$-a.e. Then $T$ has a fixed point $f$ such that $f_{0} \leq f$ (resp. $\left.f \leq f_{0}\right) \rho$-a.e.

The original proof of the existence of a fixed point of asymptotically nonexpansive mappings was not constructive. It was Shu [18] who considered a modified Mann iteration to generate an approximate fixed point sequence for such mappings. While studying asymptotically nonexpansive mappings, Schu modified the Mann iteration by

$$
x_{n+1}=t_{n} T^{n}\left(x_{n}\right)+\left(1-t_{n}\right) x_{n}
$$

for $t_{n} \in[0,1]$ and $n \in \mathbb{N}$. Schu used the iterate $T^{n}$ because it is becoming almost nonexpansive. In the investigation of monotone mappings, it is unknown whether Schu's iteration sequence generates a sequence which is monotone provided $x_{0}$ and $T\left(x_{0}\right)$ are comparable. A very important fact when investigating the existence of fixed points of such mappings. This problem forced the authors in [16] to modify Schu's iteration sequence by using the Fibonacci sequence $\{\phi(n)\}$ defined by

$$
\phi(0)=\phi(1)=1, \text { and } \phi(n+1)=\phi(n)+\phi(n-1),
$$

for any $n \geq 1$. The Fibonacci-Mann iteration [16] ((FMI) in short) is defined by

$$
h_{n+1}=\alpha_{n} T^{\phi(n)}\left(h_{n}\right)+\left(1-\alpha_{n}\right) h_{n}
$$

for $\alpha_{n} \in[0,1]$ and $n \in \mathbb{N}$. This new iteration scheme allowed the authors of [16] to prove the main results of Schu [18] for M-A-N mappings defined in uniformly convex Banach spaces. This is surprising since this class of mappings may fail to be continuous.

Next we discuss the behavior of the iteration (FMI) which will generate an approximate fixed point of M-A-N mapping in MFS.

The proof of the following lemma uses solely the partial order and is similar to the original proof done in [16] in the context of Banach spaces.

Lemma 3. [16] Let $\rho$ be convex regular modular. Let $C$ be a convex nonempty subset of $L_{\rho}$. Let $T: C \rightarrow C$ be a monotone mapping. Let $h_{0} \in C$ be such that $h_{0} \leq T\left(h_{0}\right)$ (resp. $\left.T\left(h_{0}\right) \leq h_{0}\right) \rho$-a.e. Let $\left\{\alpha_{n}\right\} \subset[0,1]$. Consider the (FMI) sequence $\left\{h_{n}\right\}$ generated by $h_{0}$ and $\left\{\alpha_{n}\right\}$. Let $f \in C$ be a fixed point of $T$ such that $h_{0} \leq f$ (resp. $\left.f \leq h_{0}\right) \rho$-a.e. Then

(i) $h_{0} \leq h_{n} \leq h_{n+1} \leq T^{\phi(n)}\left(h_{n}\right) \leq f$ (resp. $\left.f \leq T^{\phi(n)}\left(h_{n}\right) \leq h_{n+1} \leq h_{n} \leq h_{0}\right) \rho$-a.e.;

(ii) $h_{0} \leq T^{\phi(n)}\left(h_{0}\right) \leq T^{\phi(n)}\left(h_{n}\right) \leq f$ (resp. $\left.f \leq T^{\phi(n)}\left(h_{n}\right) \leq T^{\phi(n)}\left(h_{0}\right) \leq h_{0}\right) \rho$-a.e.;

for any $n \in \mathbb{N}$.

The next lemma is crucial in the proof of the main results of this work.

Lemma 4. Let $\rho$ be convex regular modular. Let $C$ be a $\rho$-bounded and convex nonempty subset of $L_{\rho}$. Assume $T: C \rightarrow C$ is an $M-A-N$ mapping with the Lipschitz constants $\left\{k_{n}\right\}$ satisfying $\sum_{n=1}^{\infty}\left(k_{n}-1\right)<\infty$. Let $h_{0} \in C$ be such that $h_{0} \leq T\left(h_{0}\right)$ (resp. $\left.T\left(h_{0}\right) \leq h_{0}\right) \rho$-a.e. Let $\left\{\alpha_{n}\right\} \subset[0,1]$. Consider the (FMI) sequence $\left\{h_{n}\right\}$ generated by $h_{0}$ and $\left\{\alpha_{n}\right\}$. Let $f \in C$ be a fixed point of $T$ such that $h_{0} \leq f$ (resp. $\left.f \leq h_{0}\right) \rho$-a.e. Then $\lim _{n \rightarrow \infty} \rho\left(h_{n}-f\right)$ exists.

Proof. Without loss of generality, assume that $h_{0} \leq T\left(h_{0}\right) \rho$-a.e. Note that, since $C$ is $\rho$-bounded, we must have $\limsup _{m \rightarrow \infty} \rho\left(h_{m}-f\right) \leq \delta_{\rho}(C)<+\infty$. From the definition of $\left\{h_{n}\right\}$, we have 


$$
\begin{aligned}
\rho\left(h_{n+1}-f\right) & \leq \alpha_{n} \rho\left(T^{\phi(n)}\left(h_{n}\right)-f\right)+\left(1-\alpha_{n}\right) \rho\left(h_{n}-f\right) \\
& =\alpha_{n} \rho\left(T^{\phi(n)}\left(h_{n}\right)-T^{\phi(n)}(f)\right)+\left(1-\alpha_{n}\right) \rho\left(h_{n}-f\right) \\
& \leq \alpha_{n} k_{\phi(n)} \rho\left(h_{n}-f\right)+\left(1-\alpha_{n}\right) \rho\left(h_{n}-f\right) \\
& =\alpha_{n}\left(k_{\phi(n)}-1\right) \rho\left(h_{n}-f\right)+\rho\left(h_{n}-f\right) \\
& \leq\left(k_{\phi(n)}-1\right) \rho\left(h_{n}-f\right)+\rho\left(h_{n}-f\right)
\end{aligned}
$$

for any $n \in \mathbb{N}$, where we used the fact that $f$ is a fixed point of $T$, the definition of the Lipschitz constants $\left\{k_{n}\right\}$ and $\left\{\alpha_{n}\right\} \subset[0,1]$. Hence

$$
\rho\left(h_{n+1}-f\right)-\rho\left(h_{n}-f\right) \leq\left(k_{\phi(n)}-1\right) \delta_{\rho}(C),
$$

for any $n \in \mathbb{N}$, which implies

$$
\rho\left(h_{n+m}-f\right)-\rho\left(h_{n}-f\right) \leq \delta_{\rho}(C) \sum_{i=0}^{m}\left(k_{\phi(n+i)}-1\right),
$$

for any $n, m \geq 1$. Let us rewrite this inequality as

$$
\rho\left(h_{n+m}-f\right) \leq \rho\left(h_{n}-f\right)+\delta_{\rho}(C) \sum_{i=0}^{m}\left(k_{\phi(n+i)}-1\right),
$$

for any $n, m \geq 1$. Next, we let $m \rightarrow \infty$ to obtain

$$
\limsup _{m \rightarrow \infty} \rho\left(h_{m}-f\right) \leq \rho\left(h_{n}-f\right)+\delta_{\rho}(C) \sum_{i=n}^{\infty}\left(k_{\phi(i)}-1\right) \leq \rho\left(h_{n}-f\right)+\delta_{\rho}(C) \sum_{i=n}^{\infty}\left(k_{i}-1\right),
$$

for any $n \geq 1$. Finally if we let $n \rightarrow \infty$, we have

$$
\limsup _{m \rightarrow \infty} \rho\left(h_{m}-f\right) \leq \liminf _{n \rightarrow \infty} \rho\left(h_{n}-f\right)+\delta_{\rho}(C) \liminf _{n \rightarrow \infty} \sum_{i=n}^{\infty}\left(k_{i}-1\right)=\liminf _{n \rightarrow \infty} \rho\left(h_{n}-f\right),
$$

since the series $\sum_{n=1}^{\infty}\left(k_{n}-1\right)$ is convergent. Therefore, we have

$$
\limsup _{m \rightarrow \infty} \rho\left(h_{m}-f\right)=\liminf _{n \rightarrow \infty} \rho\left(h_{n}-f\right),
$$

which implies the desired conclusion.

\section{Main Results}

The next result shows that the sequence generated by (FMI) has an approximate fixed point behavior which is crucial throughout.

Proposition 1. Let $\rho$ be $(U U C)$ convex regular modular. Let $C \subset L_{\rho}$ be a $\rho$-bounded $\rho$-closed convex nonempty subset. Let $T: C \rightarrow C$ be an $M-A-N$ mapping with the associated constants $\left\{k_{n}\right\}$ satisfying $\sum_{n=1}^{\infty}\left(k_{n}-1\right)<\infty$. Let $h_{0} \in C$ be such that $h_{0} \leq T\left(h_{0}\right)$ (resp. $\left.T\left(h_{0}\right) \leq h_{0}\right) \rho$-a.e. Let $f \in C$ be a fixed point of $T$ such that $h_{0} \leq f$ (resp. $\left.f \leq h_{0}\right) \rho$-a.e. Let $\left\{\alpha_{n}\right\} \subset[a, b]$, with $0<a \leq b<1$. Consider the (FMI) sequence $\left\{h_{n}\right\}$ generated by $h_{0}$ and $\left\{\alpha_{n}\right\}$. Then

$$
\lim _{n \rightarrow \infty} \rho\left(h_{n}-T^{\phi(n)}\left(h_{n}\right)\right)=0 .
$$

Proof. Without loss of generality, we assume $h_{0} \leq T\left(h_{0}\right) \rho$-a.e. From Lemma 3, we know that $h_{n} \leq h_{n+1} \leq f \rho$-a.e. Using Remark 1 , we have $\rho\left(f-h_{n+1}\right) \leq \rho\left(f-h_{n}\right)$, for any $n \in \mathbb{N}$, i.e., $\left\{\rho\left(f-h_{n}\right)\right\}$ is a decreasing sequence of positive numbers. Hence $R=\lim _{n \rightarrow \infty} \rho\left(h_{n}-f\right)$ exists. Assume 
that $R=0$, i.e., $\left\{h_{n}\right\} \rho$-converges to $f$. From Lemma 3, we obtain $h_{n} \leq T^{\phi(n)}\left(h_{n}\right) \leq f \rho$-a.e., which implies

$$
\rho\left(T^{\phi(n)}\left(h_{n}\right)-h_{n}\right) \leq \rho\left(f-h_{n}\right)
$$

for any $n \in \mathbb{N}$. Hence, we have $\lim _{n \rightarrow \infty} \rho\left(T^{\phi(n)}\left(h_{n}\right)-h_{n}\right)=0$. Next, we assume $R>0$. We have

$$
\begin{aligned}
\limsup _{n \rightarrow \infty} \rho\left(T^{\phi(n)}\left(h_{n}\right)-f\right) & =\limsup _{n \rightarrow \infty} \rho\left(T^{\phi(n)}\left(h_{n}\right)-T^{\phi(n)}(f)\right) \\
& \leq \limsup _{n \rightarrow \infty} k_{\phi(n)} \rho\left(h_{n}-f\right) \\
& =R,
\end{aligned}
$$

since $\lim _{n \rightarrow \infty} k_{n}=1$ and $f$ is a fixed point of $T$. On the other hand, we have $\rho\left(h_{n+1}-f\right) \leq$ $\alpha_{n} \rho\left(T^{\phi(n)}\left(h_{n}\right)-f\right)+\left(1-\alpha_{n}\right) \rho\left(h_{n}-f\right)$, for any $n \geq 1$. Let $\mathcal{U}$ be a non-trivial ultrafilter over $\mathbb{N}$. We have

$$
R=\lim _{\mathcal{U}} \rho\left(h_{n+1}-f\right) \leq \alpha \lim _{\mathcal{U}} \rho\left(T^{\phi(n)}\left(h_{n}\right)-f\right)+(1-\alpha) R,
$$

with $\lim _{\mathcal{U}} \alpha_{n}=\alpha \in[a, b]$. Since $\alpha \neq 0$, we have $\lim _{\mathcal{U}} \rho\left(T^{\phi(n)}\left(h_{n}\right)-f\right) \geq R$. Since $\mathcal{U}$ was an arbitrary ultrafilter, we have

$$
R \leq \liminf _{n \rightarrow \infty} \rho\left(T^{\phi(n)}\left(h_{n}\right)-f\right) \leq \limsup _{n \rightarrow \infty} \rho\left(T^{\phi(n)}\left(h_{n}\right)-f\right) \leq R
$$

Therefore, $\lim _{n \rightarrow \infty} \rho\left(T^{\phi(n)}\left(h_{n}\right)-f\right)=R$. Since

$$
\lim _{n \rightarrow \infty} \rho\left(\alpha_{n} T^{\phi(n)}\left(h_{n}\right)+\left(1-\alpha_{n}\right) h_{n}-f\right)=\lim _{n \rightarrow \infty} \rho\left(h_{n+1}-f\right)=R,
$$

and $\rho$ is $(U U C)$, then, by using Lemma 1 , we conclude that

$$
\lim _{n \rightarrow \infty} \rho\left(h_{n}-T^{\phi(n)}\left(h_{n}\right)\right)=0
$$

which completes the proof of our claim.

Recall that the map $T: C \rightarrow C$ is said to be $\rho$-compact if $\left\{T\left(f_{n}\right)\right\}$ has a $\rho$-convergent subsequence for any sequence $\left\{f_{n}\right\}$ in $C$. The following result is the monotone version of Theorem 2.2 of [18].

Theorem 5. Let $\rho$ be (UUC) convex regular modular. Let $C \subset L_{\rho}$ be a $\rho$-bounded and $\rho$-closed convex nonempty subset of $L_{\rho}$. Let $T: C \rightarrow C$ be an $M-A-N$ mapping with the Lipschitz constants $\left\{k_{n}\right\}$. Assume that $T^{m}$ is $\rho$-compact for some $m \geq 1$. Let $h_{0} \in C$ be such that $h_{0} \leq T\left(h_{0}\right)$ (resp. $\left.T\left(h_{0}\right) \leq h_{0}\right) \rho$-a.e. Let $\left\{\alpha_{n}\right\} \subset[a, 1]$ with $0<a \leq 1$. Consider the (FMI) sequence $\left\{h_{n}\right\}$ generated by $h_{0}$ and $\left\{\alpha_{n}\right\}$. Then $\left\{h_{n}\right\}$ $\rho$-converges to a fixed point $f$ of $T$ such that $h_{0} \leq f$ (resp. $\left.f \leq h_{0}\right) \rho$-a.e.

Proof. Without loss of generality, we assume $h_{0} \leq T\left(h_{0}\right) \rho$-a.e. Since $T$ is monotone, the sequence $\left\{T^{n}\left(h_{0}\right)\right\}$ is monotone increasing. Since $T^{m}$ is $\rho$-compact, there exists a subsequence $\left\{T^{\varphi(n)}\left(h_{0}\right)\right\}$ which $\rho$-converges to $f \in C$. Let us show that $\left\{T^{n}\left(h_{0}\right)\right\} \rho$-converges to $f$ and $f$ is a fixed point of $T$. Using the properties of the $\rho$-a.e. partial order, we have $T^{n}\left(h_{0}\right) \leq f \rho$-a.e., for any $n \in \mathbb{N}$. In particular, we have

$$
T^{\varphi(n)}\left(h_{0}\right) \leq T^{\varphi(n)+1}\left(h_{0}\right) \leq f \quad \rho-a . e .
$$

for any $n \in \mathbb{N}$. Using Remark 1 , we have

$$
\rho\left(f-T^{\varphi(n)+1}\left(h_{0}\right)\right) \leq \rho\left(f-T^{\varphi(n)}\left(h_{0}\right)\right),
$$


for any $n \in \mathbb{N}$. This will imply $\left\{T^{\varphi(n)+1}\left(h_{0}\right)\right\} \rho$-converges to $f$. But

$$
\rho\left(T(f)-T^{\varphi(n)+1}\left(h_{0}\right)\right) \leq k_{1} \rho\left(f-T^{\varphi(n)}\left(h_{0}\right)\right),
$$

for any $n \in \mathbb{N}$, which implies that $\left\{T^{\varphi(n)+1}\left(h_{0}\right)\right\} \rho$-converges to $T(f)$ as well, which implies $T(f)=f$ from the uniqueness of the $\rho$-limit. It is clear from the properties of the modular $\rho$, that $\left\{\rho\left(f-T^{n}\left(h_{0}\right)\right)\right\}$ is a decreasing sequence of positive real numbers. Hence,

$$
\lim _{n \rightarrow \infty} \rho\left(f-T^{n}\left(h_{0}\right)\right)=\lim _{n \rightarrow \infty} \rho\left(f-T^{\varphi(n)}\left(h_{0}\right)\right)=0,
$$

i.e., $\left\{T^{n}\left(h_{0}\right)\right\} \rho$-converges to $f$. Let us finish the proof of Theorem 5 by showing that $\left\{h_{n}\right\} \rho$-converges to $f$. Since $f$ is a fixed point of $T$, which satisfies $h_{0} \leq f$, then Lemma 3 implies $T^{\phi(n)}\left(h_{0}\right) \leq T^{\phi(n)}\left(h_{n}\right) \leq$ $f \rho$-a.e., which implies

$$
\rho\left(f-T^{\phi(n)}\left(h_{n}\right)\right) \leq \rho\left(f-T^{\phi(n)}\left(h_{0}\right)\right),
$$

for any $n \in \mathbb{N}$. Hence $\left\{T^{\phi(n)}\left(h_{n}\right)\right\} \rho$-converges to $f$. Since $\left\{h_{n}\right\}$ is monotone increasing and bounded above by $f$, we know that $\left\{\rho\left(f-h_{n}\right)\right\}$ is a decreasing sequence of positive real numbers. Hence, $\lim _{n \rightarrow \infty} \rho\left(f-h_{n}\right)=R$ exists. Let us prove that $R=0$. Let $\mathcal{U}$ be a non-trivial ultrafilter over $\mathbb{N}$. Using the definition of $\left\{h_{n}\right\}$, we have

$$
\rho\left(h_{n+1}-f\right) \leq \alpha_{n} \rho\left(T^{\phi(n)}\left(h_{n}\right)-f\right)+\left(1-\alpha_{n}\right) \rho\left(h_{n}-f\right)
$$

for any $n \in \mathbb{N}$. If we set $\lim _{\mathcal{U}} \alpha_{n}=\alpha \in[a, 1]$, we get

$$
\lim _{\mathcal{U}} \rho\left(h_{n+1}-f\right) \leq \alpha \lim _{\mathcal{U}} \rho\left(T^{\phi(n)}\left(h_{n}\right)-f\right)+(1-\alpha) \lim _{\mathcal{U}} \rho\left(h_{n}-f\right) .
$$

Since $\lim _{\mathcal{U}} \rho\left(h_{n+1}-f\right)=\lim _{\mathcal{U}} \rho\left(h_{n}-f\right)=R$, and $\lim _{\mathcal{U}} \rho\left(T^{n}\left(h_{n}\right)-f\right)=0$, we get $R \leq(1-\alpha) R$. Since $\alpha \neq 0$, we conclude that $R=0$, i.e., $\left\{h_{n}\right\} \rho$-converges to $f$.

Before we investigate a weaker convergence of the (FMI) sequence, we will need the following result, which may be seen as similar to the classical Opial condition [19]. First, we recall that a subset $C$ of $L_{\rho}$ is $\rho$-a.e.-compact if any sequence $\left\{f_{n}\right\}$ in $C$ has a $\rho$-a.e.-convergent subsequence and its $\rho$-a.e.-limit is in $C$.

Proposition 2. Let $C \subset L_{\rho}$ be a $\rho$-a.e.-compact and $\rho$-bounded convex nonempty subset of $L_{\rho}$. Let $\left\{f_{n}\right\}$ be a monotone increasing (resp. decreasing) bounded sequence in C. Set $C_{\infty}=\left\{h \in C ; f_{n} \preceq h\right.$ (resp. $h \preceq f_{n}$ ) for any $n \in \mathbb{N}\}$. Consider the $\rho$-type function $\varphi: C_{\infty} \rightarrow[0,+\infty)$ defined by

$$
\varphi(h)=\lim _{n \rightarrow \infty} \rho\left(f_{n}-h\right) .
$$

Then $\left\{f_{n}\right\}$ is $\rho$-a.e. convergent to $f \in C_{\infty}$ and

$$
\varphi(f)=\inf \left\{\varphi(h) ; h \in C_{\infty}\right\} .
$$

Moreover, if $\rho$ is (UUC), then any minimizing sequence $\left\{h_{n}\right\}$ of $\varphi$ in $C_{\infty} \rho$-converges to $f$. In particular, $\varphi$ has a unique minimum point in $C_{\infty}$.

Proof. Without loss of generality, assume that $\left\{f_{n}\right\}$ is monotone increasing. Since $C$ is $\rho$-a.e.-compact, there exists a subsequence $\left\{f_{\psi(n)}\right\}$, which is $\rho$-a.e. convergent to some $f \in C$. Using Theorem 2, we conclude that $f_{n} \leq f \rho$-a.e., for any $n \in \mathbb{N}$. Hence, $f \in C_{\infty}$, which implies that $C_{\infty}$ is nonempty. Let $h \in C_{\infty}$. Then the sequence $\left\{\rho\left(h-f_{n}\right)\right\}$ is a decreasing sequence of finite positive numbers since $C$ is $\rho$-bounded. Hence $\varphi(h)=\lim _{n \rightarrow \infty} \rho\left(f_{n}-h\right)$ exists. As we saw before, there exists a subsequence 
$\left\{f_{\psi(n)}\right\}$ of $\left\{f_{n}\right\}$, which $\rho$-a.e.-converges to $f \in C_{\infty}$. Let us prove that $\left\{f_{n}\right\} \rho$-a.e.-converges to $f$. Indeed, for any $n \geq \psi(0)$, there exists a unique $k_{n} \in \mathbb{N}$ such that $\psi\left(k_{n}\right) \leq n<\psi\left(k_{n}+1\right)$. Clearly, we have $k_{n} \rightarrow \infty$ when $n \rightarrow \infty$. Moreover, we have $f_{\psi\left(k_{n}\right)} \leq f_{n} \leq f$, for any $n \in \mathbb{N}$. Since $\left\{f_{\psi\left(k_{n}\right)}\right\} \rho$-a.e. converges to $f$, we conclude that $\left\{f_{n}\right\}$ also $\rho$-a.e. converges to $f$. Next let $h \in C_{\infty}$. Then we must have $f_{n} \leq f \leq h \rho$-a.e., which implies

$$
\rho\left(f-f_{n}\right) \leq \rho\left(h-f_{n}\right),
$$

for any $n \in \mathbb{N}$. Hence, $\varphi(f) \leq \varphi(h)$, i.e.,

$$
\varphi(f)=\inf \left\{\varphi(h) ; h \in C_{\infty}\right\} .
$$

The last part of Proposition 2 is a classical result which may be found in [14].

Now we are ready to state a modular monotone version of Theorem 2.1 of [18].

Theorem 6. Let $\rho$ be $(U U C)$ convex regular modular. Let $C \subset L_{\rho}$ be a $\rho$-a.e.-compact and $\rho$-bounded convex nonempty subset of $L_{\rho}$. Let $T: C \rightarrow C$ be an $M-A-N$ mapping with the Lipschitz constants $\left\{k_{n}\right\}$. Assume that $\sum_{n=1}^{\infty}\left(k_{n}-1\right)<\infty$. Let $h_{0} \in C$ be such that $h_{0}$ and $T\left(h_{0}\right)$ are $\rho$-a.e.-comparable. Let $\left\{\alpha_{n}\right\} \subset[a, b]$ with $0<a \leq b<1$. Consider the (FMI) sequence $\left\{h_{n}\right\}$ generated by $h_{0}$ and $\left\{\alpha_{n}\right\}$. Then $\left\{h_{n}\right\}$ is $\rho$-a.e.-convergent. Its $\rho$-a.e.-limit is a fixed point of $T \rho$-a.e.-comparable to $h_{0}$.

Proof. Without loss of generality, assume that $h_{0} \preceq T\left(h_{0}\right) \rho$-a.e. In this case, we know that $\left\{T^{n}\left(h_{0}\right)\right\}$ is monotone increasing. Proposition 2 implies that $\left\{T^{n}\left(h_{0}\right)\right\}$ is $\rho$-a.e.-convergent to $f \in C_{\infty}$ with

$$
C_{\infty}=\left\{h \in C ; T^{n}\left(h_{0}\right) \leq h \rho-a . e . \text { for any } n \in \mathbb{N}\right\} .
$$

Since $\rho$ is $(U U C), f$ is the unique minimum point of the $\rho$-type $\varphi: C_{\infty} \rightarrow[0,+\infty)$ defined by

$$
\varphi(h)=\lim _{n \rightarrow \infty} \rho\left(T^{n}\left(h_{0}\right)-h\right) .
$$

By definition of $\left\{k_{n}\right\}$, we get

$$
\varphi(f) \leq \varphi\left(T^{m}(f)\right) \leq k_{m} \varphi(f)
$$

for any $m \geq 1$. Hence $\left\{T^{m}(f)\right\}$ is a minimizing sequence of $\varphi$ since $\lim _{m \rightarrow \infty} k_{m}=1$. Using Proposition 2, we conclude that $\left\{T^{m}(f)\right\} \rho$-converges to $f$. Note that since $T^{n}\left(h_{0}\right) \leq f \rho$-a.e., we get $T^{n+1}\left(h_{0}\right) \leq T(f)$ $\rho$-a.e., for any $n \in \mathbb{N}$, which implies $f \leq T(f) \rho$-a.e., for any $n \in \mathbb{N}$. Hence $\left\{T^{m}(f)\right\}$ is monotone increasing and $\rho$-converges to $f$, which implies $T^{m}(f) \leq f \rho$-a.e. Hence $T(f)=f$ holds, i.e., $f$ is a fixed point of $T$. Using Lemma 3 , we have

$$
T^{\phi(n)}\left(h_{0}\right) \leq T^{\phi(n)}\left(h_{n}\right) \leq f \rho-\text { a.e. }
$$

for any $n \in \mathbb{N}$, which implies that $\left\{T^{\phi(n)}\left(h_{n}\right)\right\}$ also $\rho$-a.e.-converges to $f$. Proposition 1 implies

$$
\lim _{n \rightarrow \infty} \rho\left(h_{n}-T^{\phi(n)}\left(h_{n}\right)\right)=0 .
$$

Using the properties of $\rho$-convergence and $\rho$-a.e.-convergence [4], there exists a sequence of increasing integers $\left\{j_{n}\right\}$ such that $\left\{h_{j_{n}}-T^{\phi\left(j_{n}\right)}\left(h_{j_{n}}\right)\right\} \rho$-a.e.-converges to 0 . Therefore, we must have $\left\{h_{j_{n}}\right\} \rho$-a.e.-converges to $f$. Since $\left\{h_{n}\right\}$ is monotone increasing and $h_{n} \leq f \rho$-a.e., we conclude that $\left\{h_{n}\right\} \rho$-a.e.-converges to $f$. This completes the proof of Theorem 6 by noting that $f$ is a fixed point of $T$ and $h_{0} \leq f \rho$-a.e. 
Author Contributions: All authors have read and approved the final manuscript.

Funding: This work was supported by the Deanship of Scientific Research (DSR), King Abdulaziz University, Jeddah, under Grant No. (D-075-363-1438). The authors, therefore, gratefully acknowledge the DSR technical and financial support.

Acknowledgments: The authors would like to thank the anonymous referee for his/her comments that helped us improve this article.

Conflicts of Interest: The authors declare no conflict of interest.

\section{References}

1. Kozlowski, W.M. Modular Function Spaces; Series of Monographs and Textbooks in Pure and Applied Mathematics; Dekker: New York, NY, USA; Basel, Switzerland, 1988; Volume 122.

2. Diening, L.; Harjulehto, P.; Hästö, P.; Ruzicka, M. Lebesgue and Sobolev Spaces with Variable Exponents; Springer Lecture Notes; Springer: Berlin, Germany, 2011; Volume 2017.

3. Khamsi, M.A.; Kozlowski, W.M.; Reich, S. Fixed point theory in modular function spaces. Nonlinear Anal. 1990, 14, 935-953. [CrossRef]

4. Khamsi, M.A.; Kozlowski, W.M. Fixed Point Theory in Modular Function Spaces; Birkhauser: New York, NY, USA, 2015.

5. Ran, A.C.M.; Reurings, M.C.B. A fixed point theorem in partially ordered sets and some applications to matrix equations. Proc. Am. Math. Soc. 2004, 132, 1435-1443. [CrossRef]

6. Carl, S.; Heikkila, S. Fixed Point Theory in Ordered Sets and Applications: From Differential and Integral Equations to Game Theory; Springer: Berlin, Germany; New York, NY, USA, 2011.

7. Marin, M. An approach of a heat-flux dependent theory for micropolar porous media. Meccanica 2016, 51, 1127-1133. [CrossRef]

8. Khamsi, M.A.; Kirk, W.A. An Introduction to Metric Spaces and Fixed Point Theory; John Wiley: New York, NY, USA, 2001.

9. Birnbaum, Z.; Orlicz, W. Uber die Verallgemeinerung des Begriffes der zueinander konjugierten Potenzen. Studia Math. 1931, 3, 1-67. [CrossRef]

10. Khamsi, M.A.; Kozlowski, W.M.; Shutao, C. Some geometrical properties and fixed point theorems in Orlicz spaces. J. Math. Anal. Appl. 1991, 155, 393-412. [CrossRef]

11. Shutao, C. Geometry of Orlicz Spaces; Instytut Matematyczny Polskiej Akademi Nauk: Warszawa, Poland, 1996.

12. Krasnosel'skii, M.A.; Rutickii, Y.B. Convex Functions and Orlicz Spaces; P. Nordhoff Ltd.: Groningen, The Netherlands, 1961.

13. Milnes, H.W. Convexity of Orlicz Spaces. Pac. J. Math. 1957, 7, 1451-1486. [CrossRef]

14. Khamsi, M.A.; Kozlowski, W.M. On Asymptotic Pointwise Nonexpansive Mappings in Modular Function Spaces. J. Math. Anal. Appl. 2011, 380, 697-708. [CrossRef]

15. Goebel, K.; Kirk, W.A. A fixed point theorem for asymptotically nonexpansive mappings. Proc. Am. Math. Soc. 1972, 35, 171-174. [CrossRef]

16. Alfuraidan, M.R.; Khamsi, M.A. Fibonacci-Mann iteration for monotone asymptotically nonexpansive mappings. Bull. Aust. Math. Soc. 2017, 96, 307-316. [CrossRef]

17. Alfuraidan, M.R.; Bachar, M.; Khamsi, M.A. Fixed points of monotone asymptotically nonexpansive mappings in modular function spaces. J. Nonlinear Convex Anal. 2017, 18, 565-573.

18. Schu, J. Weak and strong convergence to fixed points of asymptotically nonexpansive mappings. Bull. Austral. Math. Soc. 1991, 18, 153-159. [CrossRef]

19. Opial, Z. Weak convergence of the sequence of successive approximations for nonexpansive mappings. Bull. Am. Math. Soc. 1967, 73, 591-597. [CrossRef]

(C) 2018 by the authors. Licensee MDPI, Basel, Switzerland. This article is an open access article distributed under the terms and conditions of the Creative Commons Attribution (CC BY) license (http://creativecommons.org/licenses/by/4.0/). 